\title{
Sorgen, Einstellungen, Wissen, Handeln: Die Ergebnisse des Jugendmedienschutz-Index aus medienpolitischer Sicht
}

\author{
Stephan Dreyer
}

In einer quantitativ angelegten Untersuchung hat der »JugendmedienschutzIndex« im Winter 2017 erstmals übergreifende Erkenntnisse über Sorgen, Einstellungen, Wissen und Handeln von Eltern und ihren Kindern im Jugendmedienschutz vorgestellt. Die im Auftrag der Freilwilligen Selbstkontrolle Multimedia-Diensteanbieter e.V. (FSM) erfolgte Studie vom Hans-Bredow-Institut und dem JFF - Institut für Medienpädagogik in Forschung und Praxis hatte dafür 805 Kinder und Jugendliche zwischen 9 und 16 Jahren sowie jeweils einen Elternteil befragt. Durch die verschiedenen Dimensionen und die Möglichkeit der Eltern-Kind-übergreifenden Auswertungen ermöglicht die Studie erstmals einen umfassenden, repräsentativen Einblick in das praktische Funktionieren von Jugendmedienschutz und Medienerziehung. Der Beitrag gibt einen Überblick über die zentralen Ergebnisse und ordnet diese in die aktuellen jugendmedienschutzpolitischen Debatten ein.

\section{Die Sorgen der Eltern verändern sich mit dem Alter der Kinder}

Mit Blick auf die von Eltern und Kindern genannten Sorgen bei der Online-Nutzung von Kindern treten zwei Dinge besonders hervor: Zum einen geben große Teile von Eltern und Minderjährigen an, dass sie derzeit nicht besorgt sind (Eltern: $27 \%$; Kinder: $42 \%)$. Die Gründe dafür sind unterschiedlich, öfter genannt wird aber die Einschätzung, die Risiken zu kennen und sich schützen zu können. Zum anderen weisen die von den übrigen Befragten am häufigsten genannten Sorgen eine Verschiebung auf, die mit dem Alter des jeweiligen Kindes zu tun hat. Während inhaltsbezogene Risiken mit zunehmendem Alter des Kindes von den Eltern weniger genannt werden (9-10: $36 \%$; 11-12: $38 \%$; 13-14: $20 \%$; 15-16: $16 \%$, steigen die wahrgenommenen Kontaktrisiken in der gleichen Zeit deutlich (9-10: $27 \%$; 11-12: $38 \%$; 13-14: $33 \%$; 15-16: $37 \%)$. Mit der zunehmend autonomen Mediennutzung durch ältere Kinder schätzen deren Eltern auch die zeitlichen Risiken (9-10: $6 \%$; 11-12: $10 \%$; 13-14: $15 \%$; 15-16: $15 \%)$ sowie persönliche Konsequenzen der Mediennutzung wie z.B. Viren oder urheberrechtliche Abmahnungen (9-10: $18 \%$; 11-12: $27 \%$; 13-14: $26 \%$; 15-16: $40 \%$ ) als vermehrt relevant ein.
Dieses Ergebnis spiegelt grundsätzlich die seit Jahren erkennbare Ausweitung und Ausdifferenzierung von Risiken in der Onlinenutzung Minderjähriger wider: Mit der Nutzung interaktiver Angebote und Dienste gehen auch neue Formen von Kommunikations- und Interaktionsrisiken einher. Und durch (mobile) Endgeräte, die autonom und weiter außerhalb des unmittelbaren Einflussbereiches der Eltern genutzt werden, ergeben sich zeitliche Nutzungsrisiken. ${ }^{1}$ Gut zu erkennen ist anhand des Jugendmedienschutz-Index aber, wie sich diese neuen Sorgen insbesondere bei den Eltern größerer Kinder und Jugendlicher in den Fokus schieben. Der Schutzzweck traditionellen Jugendmedienschutzes, d.h. das Inkontaktkommen mit schädlichen Inhalten statischer Medienprodukte, tritt so in den Hinter-

Stephan Dreyer ist Senior Researcher für Medienrecht und Media Governance am Hans-Bredow-Institut für Medienforschung. Daneben ist er juristischer Sprecher des unabhängigen Beschwerdeausschusses der FSM.

grund. Die Studie zeigt hier, dass dieser Umstand nicht bedeuten muss, dass klassischer Jugendschutz an den Alltagssorgen von Eltern und Kindern vorbei reguliert, sondern dass dieses Ergebnis auch bereits Ergebnis eines erfolgreichen inhaltebezogenen Schutzansatzes sein kann. Jedenfalls festzuhalten bleibt, dass der "Sorgendruck« der Eltern nicht mehr zentral im Bereich der Medieninhalte liegt, und dass Eltern mit dem Heranwachsen des eigenen Kindes veränderte Sorgenkonstellationen äußern - und einen sich entsprechend wandelnden Beratungs- und Unterstützungsbedarf haben.

\section{Jugendmedienschutz: Breit akzeptiert, hohe Erwartungen an alle Stakeholder}

Die Akzeptanz des Jugendmedienschutzes und die damit einhergehenden $\mathrm{Zu}$ gangsbeschränkungen für Minderjährige ist bei Eltern wie bei Kindern hoch (»Schutz ist wichtiger als Zugang «: Eltern 90 \%, Kinder $72 \%)$. Auch die vom derzeitigen Rechtsrahmen vorgehaltenen Schutzinstrumente (Alterskennzeichen, technische Mittel wie Altersüberprüfung, Filtersoftware) weisen hohe Unterstützungswerte auf. Auffallend bei der Abfrage der Einstellungen sind die schwachen Ergebnisse mit Blick auf die Verfügbarkeit kinderspezifischer Angebote und Unterstützungsangebote im Falle belastender Erfahrungen.

Die für Jugendschutz von Eltern zugeschriebene Verantwortung ruht verteilt auf allen relevanten Stakeholdern. Insbesondere sehen sich die Eltern zuvörderst selbst in der Pflicht, Jugendschutz im Alltag umzusetzen (94\%), dicht gefolgt von der Medienaufsicht (82\%) und den Sozialen Netzwerken (81\%). Doch auch die übrigen Akteure erhalten hohe Werte bei der Verantwortungszuschreibung (Inhalteanbieter: $78 \%$, Videoplattformen: $75 \%$, Schule: $74 \%$, Politik: $72 \%$, App-Marktplätze: $69 \%$, Einrichtungen der freiwilligen Selbstkontrolle: 67 \%). Fragt man die Eltern allerdings nach ihrer Beurteilung, wie gut diese Institutionen ihre Verantwortung wahrnehmen, ergibt sich eine deutliche Abstufung. Sich selbst (67\%), Schulen (63\%) und Bildungseinrichtungen $(56 \%)$ bewerten Eltern merheitlich gut bei der Verantwortungsübernahme. Dagegen scheiden insbesondere die Anbieter von sozialen Netzwerken (14\%), Suchmaschinen und Instant Messengern (je $19 \%$ ) schlecht ab. Auch die Politik erfüllt ihre Verantwortung aus Sicht der Eltern nicht ordentlich (»sehr gut « oder »eher gut«: nur $16 \%)$. Hier zeigen sich deutliche Optimierungspotenziale, was die Wahrnehmung der jugendmedienschutzbezogenen Aktivitäten der Stakeholder angeht.

\section{Wissen und Handeln: Nachhaltiger Jugendmedienschutz wirkt, aber Unsicherheiten bei Hilfsangeboten}

Das bei Eltern vorhandene Wissen über den gesetzlichen Jugendmedienschutz ist in Teilen hoch. Insbesondere die Schutzziele, Schutzmaßnahmen und Akteure, die seit langem Bestand haben, treten als bekannt hervor. Es scheint angesichts dieser Ergebnisse angezeigt, vor allem auf Nachhaltigkeit bei der Regulierung und Institutionalisierung in diesem Politikfeld zu setzen: Wo Eltern und Kinder keine oder wenig Zeit haben, neue Instrumente, Maßnahmen und Akteure zu »lernen « und entsprechend das Wissen darüber begrenzt ist, können diese auch nur verringert Wirkungen im Alltag entfalten. Unsicherheiten offenbart das Elternwissen wiederum im Bereich der Beratungs- 
angebote und Anlaufstellen im Falle von Problemen: Ein Viertel der Eltern kann hier keine Antwort geben; die falschen Antworten werden fast so häufig genannt wie die richtigen. $\mathrm{Zu}$ der Problematik der aufgefächerten Risiken, die über den klassischen Jugendmedienschutz hinausgehen, kommt hier offenbar die elterliche Unsicherheit im Angesicht der Vielzahl unterschiedlicher Angebote.

Entsprechend schwach fallen die Zahlen bei der Frage aus, ob Eltern Beschwerdestellen und Hilfsangebote kennen und nutzen: Nur 29 Prozent der Eltern kennen entsprechende Anlaufstellen, und nur acht Prozent dieses knappen Drittels haben sich schon einmal an ein solches Angebot gewandt. Macht man sich - den im Prinzip sehr positiven - Umstand klar, dass aus Sicht der Kinder in erster Linie die Eltern Ansprechpartner bei Problemen sind (96\%!), bleibt festzuhalten, dass hier eine offene Flanke in der derzeitigen Schutzstruktur des Jugendmedienschutzes erkennbar wird. Die Eltern sind zentrale Erziehungs- und Vertrauenspersonen in diesem System, haben aber wenig Wissen und gefühlt wenig Möglichkeiten, sich selbst Hilfe bei der Bewältigung dieser Aufgabe zu holen.

Unabhängig davon nutzen Eltern eine ganze Reihe von Regeln im Medienalltag, um ihre Kinder vor Online-Risiken zu schützen. Setzen Eltern jüngerer Kinder vor allem auf Verbote und Orientierungen an Alterskennzeichen, spielen vor allem zeitbezogene Vorgaben bei größeren Kindern eine stark zunehmende Rolle. Bei Jugendlichen steht das Gespräch an erster Stelle. Insgesamt nimmt das elterliche Engagement im Jugendmedienschutz mit zunehmendem Alter des Kindes aber ab.

\section{Erkennbare Umsetzungsmuster im Medienalltag}

Die Studie nimmt neben den Einzeldimensionen auch übergreifende Auswertungen der Befragungsergebnisse vor, um Korrelationen zwischen Sorgen, Einstellungen, Wissen und Handeln zu erkennen. Mit Blick auf die hohe Varianz auf Seiten der Eltern in allen Dimensionen arbeitet ein Teilkapitel die häufigsten Muster der elterlichen Umsetzung von Jugendmedienschutz durch Überlagerung von Sorgen, Einstellungen und Engagement heraus. Dabei zeigt sich, dass der Elterntyp des »Besorgt-überzeugt Engagierten « mit 18 Prozent am häufigsten verteten ist: Diese Eltern machen sich zwar Sorgen um die Online-Nutzung ihrer Kinder, wissen aber um bestehende Schutzmöglichkeiten und engagieren sich entsprechend. Das andere, ebenfalls nicht seltene Extrem elterlicher Umsetzungsmuster ist bei den »Unbesorgt-skeptischen Nicht-Engagierten « zu erkennen, die in Sachen Onlinerisiken sorgenfrei sind, aber ohnehin nicht viel von Jugendmedienschutz halten und sich entsprechend wenige Übernahmen in die alltägliche Erziehungspraxis finden (17\%). Während Erstere besonders bei jüngeren Kindern vorzufinden sind, haben Letztere statistisch gesehen deutlich ältere Kinder.

\section{Relevanz der Ergebnisse für die Jugendmedienschutzpolitik}

Aus Sicht der derzeitigen Debatten in der Politik liefert der Jugendmedienschutz-Index eine ganze Reihe Anknüpfungspunkte. Die zentrale Erkenntnis und Bestätigung, dass die wahrgenommenen Online-Risiken sich thematisch verbreitern und ausweiten, hat der Jugendschutzdiskurs nicht verschlafen. Es ergeben sich hier aber große Schwierigkeiten in der Findung mehrheitsfähiger, praktikabler Steuerungsansätze. Dies beginnt mit der expliziten Ausweitung des Verständnisses, was zeitgemäßer Jugendmedienschutz ist und welche Gefährdungslagen er umfassen soll. Neue Regulierungsansätze müssen dafür auch auf Begriffsebene erfolgen - der in einem früheren Diskussionspapier aufgetauchte Begriff der »informationellen Integrität« als erweitertes Schutzziel spiegelt entsprechende Suchbewegungen wider. ${ }^{2}$ Auch der Koalitionsvertrag zwischen CDU und SPD verweist auf das erweiterte Verständnis von Jugendschutzregulierung, wenn er auf den kinderspezifischen Schutz von Daten und Persönlichkeitsrechten abstellt. ${ }^{3}$ Hier den Mittelweg zwischen begrifflicher Unbestimmtheit und erkennbaren materiellen Anforderungen und ihrer Umsetzbarkeit zu finden, wird im Zentrum der kommenden Diskussionen stehen müssen.

Ein weiterer Beitrag zur Debatte ergibt sich aus der Studie mit Blick auf die Steuerungsadressaten: Angesichts der Verantwortungszuschreibungen und der als verbesserbar wahrgenommenen Verantwortlichkeitsübernahme inbesondere bei Plattformanbietern und Politik erscheinen hier Überlegungen zu geteilter Verantwortungsübernahme vordringlich. Hier trifft Regulierung auf das allgemeine Problem der Plattformregulierung, deren Haftungsprivilegien aus dem TMG und ihre Anwendbarkeit auf (jugendmedienschutzrechtliches) Ordnungsrecht immer noch nicht abschließend geklärt sind. Selbst wenn es dann politisch opportun erschiene, die Plattformen unmittelbarer und restriktiver in die Jugendschutzregulierung einzubeziehen, bliebe das Ergebnis, dass die Eltern auch die Politik selbst in der Pflicht sehen. Danach reicht es nicht, die staatlich ggf. optimierbare Umsetzung bestehender Regelungen auf die Onlinewirtschaft abzuwälzen (siehe das Beispiel NetzDG), sondern es bedarf hier neben politischen Appellen an die Anbieterverantwortlichkeit schlicht neuer Formen der staatlichen und privaten Kooperationen bei der Optimierung von Jugendmedienschutz.

Konkrete Unterstützung aus der Studie erfahren daneben elektronische Alterskennzeichen, die von Eltern akzeptiert und als Grundlage für weitere technische Instrumente gesehen werden, die im Medienalltag helfen können. Diesen Ansatz nachhaltig auszubauen ermöglicht die im Endspurt befindliche AVMD-Richtlinie, die elektronische Alterskennzeichen als eine konvergente, medienübergreifende Möglichkeit sieht, modernen Jugendschutz umzusetzen. Dabei bliebe darauf zu achten, dass Maßnahmen und Instrumente, die auf Metadaten und automatisierten Schutz aufbauen, zuvörderst die bessere Ermächtigung der Eltern zur Umsetzung individueller Erziehungskonzepte im Blick haben und das Erziehungsrecht der Eltern nicht vorbestimmen oder überprägen. Zentraler Ansatz muss hier der Ausbau niedrigschwelliger beratender Angebote für Eltern und andere Akteure in der Medienerziehung sein.

Zuletzt zeigt die gemeinsame Befragung und Auswertung von Eltern und Kindern die Relevanz der von Kindern vorgetragenen Wahrnehmungen, Einstellungen und Erwartungen für die zukünftige Politik in diesem Feld auf: Sie geben nicht nur Impulse und Denkanstöße, sondern können mit ihrem Blick aus dem Alltag von Mediennutzung und Medienerziehung auch politische Priorisierungen ermöglichen, die Jugendmedienschutz praxisrelevant und akzeptiert werden lassen.

1 Dreyer/Hasebrink/Lampert/Schröder, Entwicklungs- und Nutzungstrends im Bereich der digitalen Medien und damit verbundene Herausforderungen für den Jugendmedienschutz. Bern 2013.

2 Kohärente Regelungen im Kinder- und Jugendmedienschutz «, Diskussionspapier (Stand: 30.09.2015), abrufbar unter https:// www.heise.de/downloads $/ 18 / 1 / 6 / 0 / 2 /$ 8/0/3/Diskussionspapier_JuSchG_AN.pdf.

3 »Ein neuer Aufbruch für Europa. Eine neue Dynamik für Deutschland. Ein neuer Zusammenhalt für unser Land «, Koalitionsvertrag zwischen CDU, CSU und SPD v. 7. Februar 2018, S. 23

Brüggen, N./Dreyer, S./Drosselmeier, M/Gebel, C.; Hasebrink, U.; Rechlitz, M. (2017): Jugendmedienschutzindex. Der Umgang mit onlinebezogenen Risiken. Ergebnisse der Befragung von Heranwachsenden und Eltern. Hrsg.: FSM - Freiwillige Selbstkontrolle Multimedia-Diensteanbieter e.V. Abrufbar unter www.fsm.de/de/jugendmedienschutzindex. 